\title{
Image segmentation based on gray-level spatial correlation maximum between-cluster variance
}

\author{
Fu Zeng ${ }^{1, a}$, He Jianfeng ${ }^{1, b^{*}}$, Xiang Yan $^{1}$,Cui Rui ${ }^{1}$, Yi Sanli ${ }^{1}$ \\ ${ }^{1}$ School of Information Engineering and Automation , Kunming \\ University of Science and Technology, Kunming 650500 \\ aemail: fuzeng2013@163.com ${ }^{b^{*}}$ email: jfenghe@qq.com
}

\begin{abstract}
.
When processing the background and target blurred image, 1D-Otsu and 2D-Otsu segmentation effect is not good. The proposed algorithm used the gray value of the pixels and their similarity with neighboring pixels in gray value to build a histogram which was called gray-level spatial correlation histogram. Then threshold value is obtained by calculating GLSC histogram maximum between-class variance. Integral figure was introduced in order to make the time complexity from original $O\left(\left(\mathrm{~N}^{2} \times \mathrm{L}\right)^{2}\right)$ to $O\left(\mathrm{~N}^{2} \times \mathrm{L}\right)$. The experimental results show that the proposed method image segmenting is better than $1 \mathrm{D}-\mathrm{Otsu}$ and 2D-Otsu, when processing the background and target blurred image.
\end{abstract}

Keywords: Otsu; gray-level spatial correlation; Integral figure; image segmentation

\section{Introduction}

Threshold segmentation is usual and efficient in image segmentation methods. Its main idea is choosing a threshold which can distinguish the image background and target in an image.

Otsu threshold method is a kind of global automatic nonparametric unsupervised algorithm threshold value, which is based on the maximum variance between measure criterion[1]. Because the 1D-Otsu algorithm only considers the gray level distribution information of the image, thus in under condition of the high signal noise ratio and the clear boundaries between the object and the background, the 1D-Otsu algorithm may get a good segmentation 
result. Conversely, the segmentation result may be poor or error [2]. Liu [3] in 1993 proposed an improved method, which uses pixel gray and the average gray level of the neighborhood to build a two-dimensional histogram, and then uses the Otsu threshold method to find the threshold. The two-dimensional Otsu algorithm has a good effect for de-noising by ignored the information of away from the main diagonal area (noise mostly distributed here)[4]. However, for some good quality image and stable background, the probability of the away from the main diagonal area is not necessarily small [5], so the two-dimensional Otsu algorithm may lead to some information loss. Because constructing histogram by one-dimensional expanded to two-dimensional, so the computational complexity will increase exponentially, which may limit the application of this method.

Xiao [6] reports a method of gray-level spatial correlation histogram (GLSC), which uses the similar neighborhood pixels to build histogram; and combines with entropy threshold method to segment image. This method can effectively extract the edge from the image. In this paper, we combine with GLSC histogram and Otsu algorithm, present a new algorithm of based on gray-level spatial correlation maximum between-cluster variance (GLSC-Otsu).

\section{Creation of a Gray-Scale Spatial Correlation Histogram}

Let $f(\mathrm{x}, \mathrm{y})$ be the gray value of the pixel located at the point $(\mathrm{x}, \mathrm{y})$ in a digital image $F=\{\mathrm{f}(\mathrm{x}, \mathrm{y}) \mid \mathrm{x} \in\{1,2, \ldots, \mathrm{Q}\}, \mathrm{y} \in\{1,2, \ldots, \mathrm{R}\}\}$ of size $Q \times R$. To construct GLSC histogram as follows: for the pixel located at the point $(\mathrm{x}, \mathrm{y})$, let $g(\mathrm{x}, \mathrm{y})$ be the number of the pixels of which the gray value is close to it in the corresponding $N \times N$ neighborhood, where $\mathrm{N}$ is a positive odd number. The $g(\mathrm{x}, \mathrm{y})$ is calculated as

$$
\begin{gathered}
g(\mathrm{x}, \mathrm{y})=\sum_{i=\frac{N-1}{2}}^{\frac{N-1}{2}} \sum_{j=\frac{N-1}{2}}^{\frac{N-1}{2}} \Im(|f(\mathrm{x}+\mathrm{i}, \mathrm{y}+\mathrm{j})-\mathrm{f}(\mathrm{x}, \mathrm{y})| \leq \zeta) \\
\text { Where, } \Im(|f(\mathrm{x}+\mathrm{i}, \mathrm{y}+\mathrm{j})-\mathrm{f}(\mathrm{x}, \mathrm{y})| \leq \zeta)= \begin{cases}1 & |f(\mathrm{x}+\mathrm{i}, \mathrm{y}+\mathrm{j})-\mathrm{f}(\mathrm{x}, \mathrm{y})| \leq \zeta \\
0 & |f(\mathrm{x}+\mathrm{i}, \mathrm{y}+\mathrm{j})-\mathrm{f}(\mathrm{x}, \mathrm{y})|>\zeta\end{cases}
\end{gathered}
$$


$\zeta$ is classified as similar to the size of the pixel value(For example, if $\zeta=3$, said $A \pm 3$ the scope of the pixel values are similar to $A$ pixels).

The GLSC histogram is constructed by using the pixel's gray value $f(\mathrm{x}, \mathrm{y})$ and $g(\mathrm{x}, \mathrm{y})$; Specific as follows

$$
p(\mathrm{k}, \mathrm{m})=P \operatorname{rob}(\mathrm{f}(\mathrm{x}, \mathrm{y})=\mathrm{k} \text { and } \mathrm{g}(\mathrm{x}, \mathrm{y})=\mathrm{m})
$$

Where $k \in\{0,1, \ldots, 255\}, m \in\{1,2, \ldots, N \times N\}$.The normalized histogram is approximated by using the formula

$$
p(\mathrm{k}, \mathrm{m})=\frac{f(\mathrm{k}, \mathrm{m})}{\mathrm{S} \times \mathrm{R}}
$$

In the formula (4), $f(\mathrm{k}, \mathrm{m})$ represents number of pixels with gray value $\mathrm{k}$ and m pixels of similar gray value in $N \times N$ neighborhood. $\mathrm{S} \times \mathrm{R}$ represents number of pixels in the image.

\section{GLSC-Otsu Segmentation Algorithm}

Supposing the image segmentation threshold is $(\mathrm{s}, \mathrm{t})$, it can be divided into $C_{0} 、 C_{1} 、 C_{2}$ and $C_{3}$ classes. They represent respectively the edge, objects, and background noise, and they have four different distributions of probability density function.

$$
\begin{aligned}
& \omega_{0}=P_{r}\left(\mathrm{C}_{0}\right)=\sum_{i=1}^{s} \sum_{j=1}^{t} p_{i j}=\omega_{0}(\mathrm{~s}, \mathrm{t}) \\
& \omega_{1}=P_{r}\left(\mathrm{C}_{1}\right)=\sum_{i=s+1}^{L} \sum_{j=t+1}^{N^{*} N} p_{i j}=\omega_{1}(\mathrm{~s}, \mathrm{t}) \\
& \omega_{2}=P_{r}\left(\mathrm{C}_{2}\right)=\sum_{i=s+1}^{L} \sum_{j=1}^{t} p_{i j}=\omega_{2}(\mathrm{~s}, \mathrm{t}) \\
& \omega_{3}=P_{r}\left(\mathrm{C}_{3}\right)=\sum_{i=1}^{s} \sum_{j=t+1}^{N^{*} N} p_{i j}=\omega_{3}(\mathrm{~s}, \mathrm{t})
\end{aligned}
$$

Four types corresponding mean vectors are

$\boldsymbol{\mu}_{0}=\left(\mu_{i 0}(\mathrm{~s}, \mathrm{t}) / \omega_{0}(\mathrm{~s}, \mathrm{t}), \mu_{j 0}(\mathrm{~s}, \mathrm{t}) / \omega_{0}(\mathrm{~s}, \mathrm{t})\right)^{T}$ 


$$
\begin{aligned}
& \boldsymbol{\mu}_{1}=\left(\mu_{i 1}(\mathrm{~s}, \mathrm{t}) / \omega_{1}(\mathrm{~s}, \mathrm{t}), \mu_{j 1}(\mathrm{~s}, \mathrm{t}) / \omega_{1}(\mathrm{~s}, \mathrm{t})\right)^{T} \\
& \boldsymbol{\mu}_{2}=\left(\mu_{i 2}(\mathrm{~s}, \mathrm{t}) / \omega_{2}(\mathrm{~s}, \mathrm{t}), \mu_{j 2}(\mathrm{~s}, \mathrm{t}) / \omega_{2}(\mathrm{~s}, \mathrm{t})\right)^{T} \\
& \boldsymbol{\mu}_{3}=\left(\mu_{i 3}(\mathrm{~s}, \mathrm{t}) / \omega_{3}(\mathrm{~s}, \mathrm{t}), \mu_{j 3}(\mathrm{~s}, \mathrm{t}) / \omega_{3}(\mathrm{~s}, \mathrm{t})\right)^{T}
\end{aligned}
$$

The total mean vector of GLSC histogram is

$\boldsymbol{\mu}_{\mathbf{T}}=\left(\mu_{T i}, \mu_{T j}\right)^{T}=\left(\sum_{i=1}^{L} \sum_{j=1}^{N^{*} N} i p_{i j}, \sum_{i=1}^{L} \sum_{j=1}^{N^{*} N} j p_{i j}\right)^{T}$

Define a class of discrete matrix as formula (14).

$$
\boldsymbol{S}_{\boldsymbol{B}}=\sum_{k=0}^{3} P_{r}\left(\mathrm{C}_{k}\right)\left[\left(\boldsymbol{\mu}_{\mathrm{k}}-\boldsymbol{\mu}_{\mathrm{T}}\right)\left(\boldsymbol{\mu}_{\mathrm{k}}-\boldsymbol{\mu}_{\mathbf{T}}\right)^{T}\right]
$$

From formulas (9) (10) (11) (12) $\mu_{0 i}=\mu_{i 0} / \omega_{0} 、 \mu_{1 i}=\mu_{i 1} / \omega_{1} 、 \mu_{2 i}=\mu_{i 2} / \omega_{2}$ 、 $\mu_{3 i}=\mu_{i 3} / \omega_{3} 、 \mu_{0 j}=\mu_{j 0} / \omega_{0} 、 \mu_{1 j}=\mu_{j 1} / \omega_{1} 、 \mu_{2 j}=\mu_{j 2} / \omega_{2}$ and $\mu_{3 j}=\mu_{j 3} / \omega_{3}$. Trace of $S_{\boldsymbol{B}}$ as a class of discrete degree of measurement as shown in formula (15)

$$
\begin{aligned}
\operatorname{tr} S_{B}= & \omega_{0}\left[\left(\frac{\mu_{i 0}}{\omega_{0}}-\mu_{T i}\right)^{2}+\left(\frac{\mu_{j 0}}{\omega_{0}}-\mu_{T j}\right)^{2}\right]+\omega_{1}\left[\left(\frac{\mu_{i 1}}{\omega_{1}}-\mu_{T i}\right)^{2}+\left(\frac{\mu_{j 1}}{\omega_{1}}-\mu_{T j}\right)^{2}\right] \\
& +\omega_{2}\left[\left(\frac{\mu_{i 2}}{\omega_{2}}-\mu_{T i}\right)^{2}+\left(\frac{\mu_{j 2}}{\omega_{2}}-\mu_{T j}\right)^{2}\right]+\omega_{3}\left[\left(\frac{\mu_{i 3}}{\omega_{3}}-\mu_{T i}\right)^{2}+\left(\frac{\mu_{j 3}}{\omega_{3}}-\mu_{T j}\right)^{2}\right]
\end{aligned}
$$

The principle of Otsu algorithm is to calculate the maximum between-cluster variance of image information. Edge is a structural boundary between object and background information, and sometimes it is difficult to identify whether the edges belongs to target or background. We believe that the edge is more important than the target and background information[6]. Based on the above analysis, regarding the GLSC histogram as an information source, elements of different $m$ in it should not be treated equally in the maximum between-cluster variance calculation as in Liu's method[3]. In this paper, the maximum between-cluster variance calculation of elements in GLSC histogram are weighted by a nonlinear function associated with $\mathrm{m}$ and $\mathrm{N}$ given by 
Weight $(\mathrm{m}, \mathrm{N})=\frac{1+e^{\frac{-9 m}{N \times N}}}{1-e^{\frac{-9 m}{N \times N}}}$. Where $\mathrm{N}$ is usually taken odd, $m \in\{1,2 \ldots \mathrm{N} \times \mathrm{N}\}$. The best threshold value $\left(\mathrm{s}^{*}, \mathrm{t}^{*}\right)$ to satisfy the following

$$
\left(\mathrm{s}^{*}, \mathrm{t}^{*}\right)=\arg \max _{1 \leq s, t \leq N^{*} N}\left\{\operatorname{tr} \mathbf{S}_{\mathbf{B}} * \operatorname{Weight}(\mathrm{m}, \mathrm{N})\right\}
$$

In order to obtain the best threshold, it needs to search in the projection of plane $N^{2} \times L$. Projection plane can be divided into four regions by each threshold. So if ignoring the extraction time of histogram, computing complexity of GLSC-Otsu segmentation algorithm is $O\left(\mathrm{~L} \times \mathrm{N}^{2}\left(\mathrm{~s} \times \mathrm{t}+(\mathrm{L}-\mathrm{s}) \times\left(\mathrm{N}^{2}-\mathrm{t}\right)+(\mathrm{L}-\mathrm{s}) \times \mathrm{t}+\mathrm{S} \times\left(\mathrm{N}^{2}-\mathrm{t}\right)\right)\right)=\mathrm{O}\left(\left(\mathrm{N}^{2} \times \mathrm{L}\right)^{2}\right)$. As $\mathrm{N}$ worth increases computational complexity exponentially, there is no doubt that the use of this method is restricted. In this paper, using the integral image method to quickly select threshold is proposed.

\section{Integral Figure Fast Selecting Threshold Method}

The concept of integral figure is put forward by Viola[7], when used to extract the image feature values. The integral image at location $(\mathrm{x}, \mathrm{y})$ contains the sum of the pixels above and to the left of $\left(x^{\prime}, y^{\prime}\right)$.It is defined as $i i(\mathrm{x}, \mathrm{y})=\sum_{x^{\prime} \leq x, y^{\prime} \leq y} i\left(\mathrm{x}^{\prime}, \mathrm{y}^{\prime}\right) \cdot$ where $i i(\mathrm{x}, \mathrm{y})$ integral figure, $i\left(\mathrm{x}^{\prime}, \mathrm{y}^{\prime}\right)$ original image.

From the formula (15) in the previous section, it needs compute $\omega_{0} 、 \omega_{1} 、 \omega_{2}$ 、 $\omega_{3} 、 \mu_{i 0} 、 \mu_{i 1} 、 \mu_{i 2} 、 \mu_{i 3} 、 \mu_{j 0} 、 \mu_{j 1} 、 \mu_{j 2} 、 \mu_{j 3} 、 \mu_{T i}$ and $\mu_{T j}$ to get $t r S_{B}$. For the same image, $\mu_{T i}$ and $\mu_{T j}$ are fixed. For each threshold $\left(s^{*}, t^{*}\right)$, if calculates class of discrete degree of measurement, It must be recalculated from $i=0, j=0$. From analysis of the previous section, its computing complexity is $\mathrm{O}\left(\left(\mathrm{N}^{2} \times \mathrm{L}\right)^{2}\right)$. In this paper, integral figure is used to quickly select the threshold.

Let GLSC histogram as $\omega$, the corresponding integral figure is $\omega_{-i i} \cdot \omega_{0}$ 、 $\omega_{1} 、 \omega_{2}$ and $\omega_{3}$ as following:

$$
\omega_{0}=\omega_{-i i}(\mathrm{~s}, \mathrm{t})
$$




$$
\begin{aligned}
& \omega_{1}=\omega_{-i i}(\mathrm{~L}, \mathrm{M})-\omega_{-i i}(\mathrm{~L}, t)-\omega_{-i i}(\mathrm{~s}, \mathrm{M})+\omega_{-i i}(\mathrm{~s}, t) \\
& \omega_{2}=\omega_{-i i}(L, \mathrm{t})-\omega_{-i i}(\mathrm{~s}, \mathrm{t}) \\
& \omega_{3}=\omega_{-i i}(\mathrm{~s}, \mathrm{M})-\omega_{-i i}(\mathrm{~s}, t)
\end{aligned}
$$

In the same way ,we can get $\mu_{i 0}, \mu_{i 1}, \mu_{i 2}, \mu_{i 3}, \mu_{j 0}, \mu_{j 1}, \mu_{j 2}$ and $\mu_{j 3}$.The probability of different regions can be calculated through the steps subtraction algorithm using the above methods. When we calculate $\mu_{i 0} 、 \mu_{i 1} 、 \mu_{i 2} 、 \mu_{i 3} 、 \mu_{j 0}$ 、 $\mu_{j 1} 、 \mu_{j 2}$ and $\mu_{j 3}$, a one-dimensional calculation first, then after addition and subtraction operation can be get. So, the computational complexity of algorithm from $L\left(\left(\mathrm{~N}^{2 *} \mathrm{~L}\right)^{2}\right)$ to $L\left(\mathrm{~N}^{2 *} \mathrm{~L}\right)$.It saves the running time and storage space.

\section{Experimental Results and Discussions}

1.1 In this experiment, Figure “Bacteria”( $364 \times 364)$ and “Block” $(244 \times 244)$ are used to verify the effectiveness of the proposed algorithm. Selection of parameters as followed: take $N=17$ 、 $\zeta=0,1,2 \ldots, 9$,then segment above images. In accordance to segmentation error rate[13], it can get the best segmentation effect, when take $\zeta=3$.In the same way, neighborhood sizes of $3 \times 3 、 5 \times 5 、 7 \times 7 、 9 \times 9 、 11 \times 11$ 、 $13 \times 13 、 15 \times 15$ and $19 \times 19$ are carried out. Finally, neighborhood sizes of $17 \times 17$ with $\zeta=3$ is the best choice.

1.2 Figure 1 shows the segmentation result of 1D-Otsu theory [1], Liu's 2D-Otsu segmentation theory[3] and the algorithm in this paper. Column 1 to 2 from left to right shows testing images and their ground-truth images, and column 3 to 5 shows segmentation results using above three methods. Table 1 shows time consumption of different methods. From figure 1, when processing the background and target blurred image, the results segmenting by the method presented in this paper is better than $1 \mathrm{D}-\mathrm{Otsu}$ and 2D-Otsu. From table 1, the algorithm runs although slower than 1D-Otsu, but a lot faster than 2D-Otsu. 

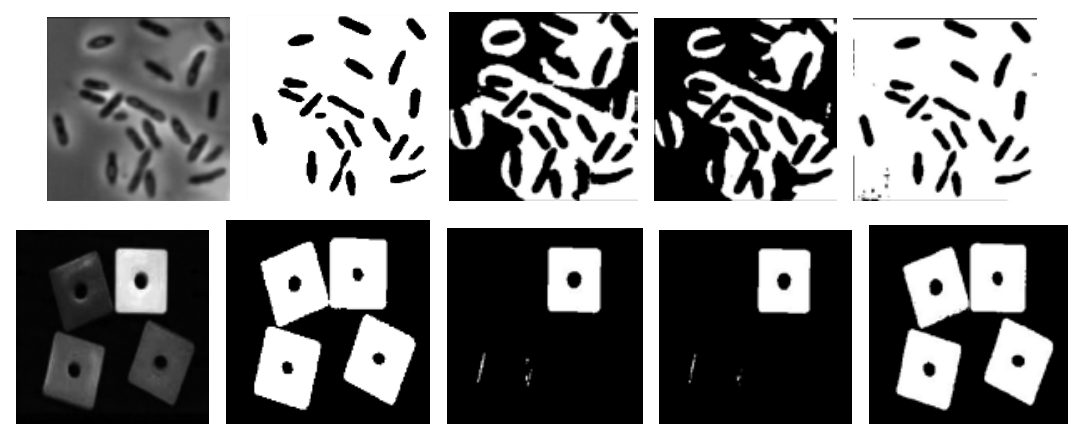

1.3 Fig 1 From left to right testing image、 ground-truth images、 segmentation results of 1D-Otsu、segmentation results of 2D-Otsu and segmentation results of our method

1.4 Table 1. Time consumption of different methods

\begin{tabular}{cccc}
\hline Image title & 1D OTSU(s) & 2D OTSU(s) & Our method(s) \\
\hline Bacteria & 0.0032 & 38.24 & 1.27 \\
Block & 0.0010 & 38.26 & 0.50 \\
\hline
\end{tabular}

\section{Conclusion}

A method for image segmentation based on gray-level spatial correlation maximum between-cluster variance is proposed. The algorithm has stronger ability to distinguish the edge than the 1D-Otsu and 2D-Otsu. In the target and background boundaries blurred, it still has a good effect. And the algorithm in terms of time consumed is much faster than the 2D-Otsu algorithm.

\section{Acknowledgement}

This study was supported by the National Natural Science Foundation of China (No.11265007), and Supported by Scientific Research Foundation for Returned Scholars, Ministry of Education of china (No. 2010-1561).

\section{References}

[1] OTSU N.A threshold selection method from gray-level histogram[J].IEEE Trans System Man and Cybernetics,1979,9(1):62-66. 
[2]Wu Y Q, Pan Z, Wu W Y. Image thresholding based on two-dimensional histogram oblique segmentation and its fast recurring algorithm[J].Journal on Communications,2008,29(4):77-89.

[3] Liu J Z, Su W Q. The automatic thresholding of gray-level picture via 2D Otsu method[J]. ACTA AUTOMATICA SINICA, 1993,19(1):101-105.

[4] Yue F, Zuo W M, Wang K Q. Decomposition Based Two-dimensional Threshold Algorithm for Gray Images[J]. ACTA AUTOMATICA SINICA, 2009,35(7):1022-1027.

[5] Chen Q, Xiong B L, Lu J. Improved Two-Dimensional Otsu Image Segmentation Method and Fast Recursive Realization[J].Journal of Electronics \& Information Technology, 2010,32(5):1100-1104.

[6] Y. Xiao, Z. Cao, S. Zhong, New entropic thresholding approach using gray-level spatial correlation histogram, Opt. Eng. 49 (12) (2010) 127007

[7] P Viola, M Jones. Rapid Object Detection using a Boosted Cascade of Simple Features, Computer Vision and Pattern Recognition, 2001, Volume 1, 8-1. 